\title{
Long Duration Linear Frequency Modulated Pulse Based Spotlight Synthetic Aperture Radar Sub-image Reconstruction Error Reduction Technique
}

\author{
Peter Manyere $^{1}$, Edward Chikuni $^{2}$, Joseph Mudare ${ }^{3}$, Thanks Marisa ${ }^{4}$, Emmanuel \\ Rashayi $^{5}$ \\ University of Zimbabwe \\ P.O.Box MP 167, Mt Pleasant \\ Harare, Zimbabe \\ \{pmanyere@eng.uz.ac.zw, echikuni@ieee.org, josephmudare@yahoo.com, \\ erashayi@eng.uz.ac.zw, thanxm@gmail.com\}
}

\begin{abstract}
The Spotlight Synthetic Aperture Radar (SSAR) is capable of producing high quality images with very high resolution in both the azimuth and range when operated with long duration pulses. Long duration pulses also allow high power to be transmitted using miniaturized radar transmitters, thus also extending the radar range. The major drawback to the use of long duration radar pulses is the need for a large radar memory and the reduction in processing speed. Segmentation of the radar data and its parallel processing addresses the limitation of memory and processing speed. However, the recombination of processed segments reduces the image quality due to recombination errors. The use of both Fast Fourier transformation (FFT) and Discrete Cosine transformation (DCT) in SSAR data processing eradicates the errors associated with sub-image reconstruction.
\end{abstract}

Keywords: Long duration pulses, Spotlight SAR, Data Segmentation, Parallel processing, Sub-image recombination, discrete cosine transformation.

\section{Introduction}

Spotlight Mode Synthetic Aperture Radar (SSAR) offers very high image geometric resolution in both the azimuth and range direction when long duration pulses are employed. This is attributable to the continuous steering of the airborne or spaceborne antenna so that the transmitted beam remains focused on the target scene of interest thereby increasing the radar viewing angle. The longer the duration of the radar pulse, the wider the bandwidth and the better image range resolution. Long duration radar pulses offer improved signal to noise ratio of the transmitted and received pulses, higher transmitted energy and extended radar range. This presents the opportunity to miniaturize radar transceivers yet producing the required transmit power. Major limitation of long duration pulse data is the need for large memory and more processing time, which slows down the SSAR processing system. Segmentation or partitioning of the received SSAR data in 1 or 2 dimension and parallel processing

ACRID 2017, June 20-21, Victoria Falls, Zimbabwe

Copyright (C) 2017

DOI 10.4108/eai.20-6-2017.2270830 
eradicates processing time challenges. In this research, 2-D segmentation is employed prior to parallel processing. The recombination of sub-images poses a challenge through errors that tend to compromise image quality. We present in this paper, a technique that can help eradicate sub-image reconstruction errors through use of both Fast Fourier transformation (FFT) and Discrete Cosine transformation (DCT).

\section{SSAR Data Generation}

The choice of a particular waveform in radar and its processing technique depends on the radar's specific mission and role [1]. The system can use either continuous wave form or pulsed waveform, with or without modulation. The modulation technique can be analog or digital. In SAR, a 2-D terrain reflectivity function can be assumed rather than a more general 3-D reflectivity function [2], [3]. For convenience sake, a rectangular shape of the transmitted LFM pulse is selected and the amplitude of the pulse is modified to $A_{o}$.

So, the radar transmits an LFM chirp signal with chirp rate $\gamma$, inter-pulse period T and centre frequency $f_{c}$. The pulses exit the transmitter with a signal $s_{t x}(n, t)$. When $\hat{t=t-n T}$ the transmitted signal becomes:

$$
\left.s_{t x}(n, t)=A_{o} \operatorname{rect}\left(\begin{array}{c}
\hat{t} \\
T_{p}
\end{array}\right) e^{j\left[2 \pi f_{c} t+\pi \gamma \hat{t}^{2}\right.}\right] .
$$

The received signal from a point scatterer at $\left(X_{t}, Y_{t}, Z_{t}\right)$ at pulse $n$ is given by:

$$
\left.\left.\left.s_{r x}(n, t)=a_{t} r e c t\left(\frac{\hat{t}-\tau}{T_{p}}\right) e^{j\left[2 \pi f_{c}(t-\tau\right.} d\right)\right] e^{j \pi \gamma(t-\tau} d\right)^{2}
$$

where $a_{t}=\sqrt{\sigma_{t}}, \sigma_{t}$ is reflectivity or radar cross-section area (RCS) and $\tau_{d}$ is the total delay time of the pulse from the transmit antenna to the target and back to the receive antenna. The total delay time can be approximated as ${ }_{\tau_{d}} \approx \frac{2 R_{t}}{c}$ where $R_{t}$ is slant range to target. The received signal can be viewed as a 2-D signal in the coordinates $n$ and $t$. The received signal becomes:

$$
s_{r x}(n, t)=a_{t} r e c t\left(\frac{\hat{t}-\frac{2 R_{t}}{c}}{T_{p}}\right) e^{j\left[2 \pi f_{c}\left(t-\frac{2 R_{t}}{c}\right)\right]} e_{e}^{j \pi \gamma\left(t-\frac{2 R_{t}}{c}\right)^{2}} .
$$

After de-ramping, the intermediate frequency (IF) is a base-band signal given by:

$$
s_{I F}(\hat{n, t)})=a_{t} r e c t\left(\frac{\hat{t}-\frac{2 R_{t}}{c}}{T_{p}}\right) e^{-j 4 \pi\left(\frac{f_{c}+\gamma \hat{t}}{c}\right)\left(R_{t}-R_{o}\right)} e^{j \frac{4 \pi \gamma}{c^{2}}\left(R_{t}^{2}-R_{o}^{2}\right)} .
$$


Or can conveniently be expressed as follows:

$$
s_{I F}(n, t)=a_{t} r e c t\left(\frac{\hat{t}-\frac{2 R_{t}}{c}}{T_{p}}\right) e^{-j \frac{4 \pi \gamma}{c}\left(\frac{f_{c}}{\gamma}+\hat{t}-\frac{2 R_{o}}{c}\right)\left(R_{t}-R_{o}\right)} e^{j \frac{4 \pi \gamma}{c^{2}}\left(R_{t}^{2}-R_{o}^{2}\right)} .
$$

The motion compensated 2-D digital signal for the received $N_{p}$ radar pulses and each pulse sampled by $\mathrm{K}$ samples can therefore be represented by:

$$
S_{d}(n, k)=a_{t} \sum_{n=0}^{N} \sum_{k=0}^{-1} K^{-1} e^{j \phi_{1}(n, k)}
$$

where;

$$
\phi_{1}(n, k)=-\frac{4 \pi \gamma}{c}\left(\frac{f_{c}}{\gamma}+\frac{k}{F_{s}}-\frac{T_{p}}{2}-\frac{T_{s}}{2}\right)\left(R_{t}-R_{0}\right)+\frac{4 \pi \gamma}{c^{2}}\left(R_{t}-R_{0}\right)^{2} .
$$

$R_{0}$ is radar antenna phase centre to scene centre; $k=$ sample number; $T_{S}=$ sampling period ; $T_{p}=$ pulse width or duration; $F_{s}=$ sampling frequency.

The last term $\frac{4 \pi \gamma}{c^{2}}\left(R_{t}-R_{0}\right)^{2}$ in (7) represents the residual video phase (RVP) responsible for the quadratic phase error [2], [4]. This unwanted phase term arises from the range dechirping process and does not positively contribute to geometric positioning or resolution of scatterers in either range or cross-range. Usually the RVP is small and can be safely ignored in image formation without any serious impact on image quality. The phase of the stabilized signal then becomes:

$$
\phi_{1}(n, k)=-\frac{4 \pi \gamma}{c}\left(\frac{f_{c}}{\gamma}+\frac{k}{F_{s}}-\frac{T_{s}}{2}-\frac{T_{p}}{2}\right)\left(R_{t}-R_{0}\right)
$$

In SSAR phase history data, signal phase is more important than magnitude. For this reason, SSAR system analysis often concentrates on the phase of the signal backscattered from a single idealized point scatterer at some location within an image scene [4]. The idea is to analyze a point target. Although the transmitted and received radar signals are real quantities, for acceptable convenience, these signals are expressed in complex exponential form. Point target analysis allows the modeling of all signal phase contributions from signal generation, transmission and reception. The model takes care of demodulation, real-time motion compensation, pre-summing and digitization. The stabilized received SSAR expression of equation 7 above was used to simulate data of size $128 \times 1989$ which was used in this research. The SSAR raw data generated is as shown in fig. 1 below. It is observed that the two images differ slightly in texture with the noiseless image being smoother than the noisy image. 


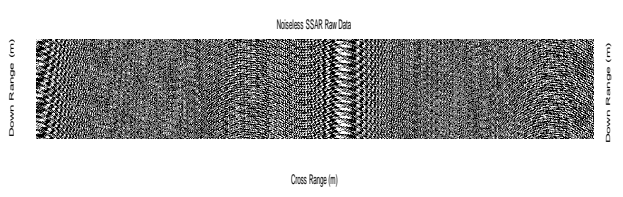

a. Noise-Free Raw Data

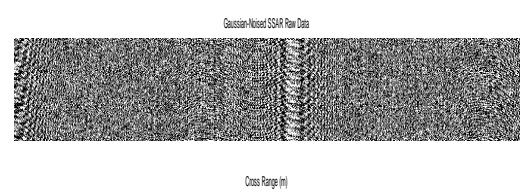

(b) Noisy Raw Data

Fig. 1. SSAR Raw Data

Fig. 2 below shows the real target locations from the simulated SSAR data in three dimension.

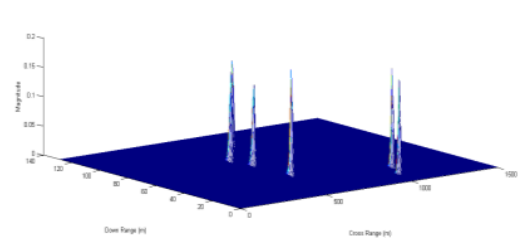

Fig.2. SSAR target locations in 3-dimension

\section{Characteristics of SSAR Data}

From the expression for the 2-D sampled and stabilized (motion compensated) SAR signal, the related phase was developed. Each sample in the data includes the quantized amplitude and phase components. Each element of the array therefore represents an image pixel with amplitude related to the strength of a backscatter coefficient in the corresponding scene area [3]. The corresponding scene center frequency is zero since it is also the reference point for motion compensation.

Each return pulse is recorded at an angle in the spatial frequency domain related to the relative rotational angle $\theta_{a}$ (aperture viewing angle or squint angle) of the scene.

Due to the quadratic nature of the motion compensated phase of the received echo signal, the 2-D phase history data is recorded in form of an annulus in $K_{x} K_{y}$ plane as shown in figure 3 below. The radial positioning of each return pulse is proportional to the instantaneous transmitted frequency $f_{c}+\hat{\gamma}$. The scatterer coordinates $X_{t}$ and $Y_{t}$ can be coded in frequency along $K_{x}$ and $K_{y}$ axes (spatial frequencies). Therefore, the polar format angle $\theta_{p}$ and the distances $R_{t}$ and $R_{a}$ vary with pulse number $n$. While $\theta_{p}$ is constant in a pulse, it varies linearly with the measured angle $\theta_{a}$ from pulse to pulse. So, the signal returned from a scatterer at $\left(X_{t}, Y_{t}, Z_{t}\right)$ for pulse $n$ is stored in the processor space along the line $\theta_{p}=\theta_{a}(n)[4]$. 
The spatial frequency $K_{c}$ corresponds to the aperture centre pulse $n_{n=n}$ returned by scene centre. To process such data, various reconstruction techniques are employed in the process. These include the Fourier transformation, Interpolation, Chirp-Z transformation and use of reconstruction filters [5], [6], [7], [8], [9], [10]. In this paper, the Fourier transformations (FFT and IFFT) were employed on each SSAR data segment for reconstruction of the composite SSAR image. In fig. 4 we show a typical SSAR data geometry in polar format.

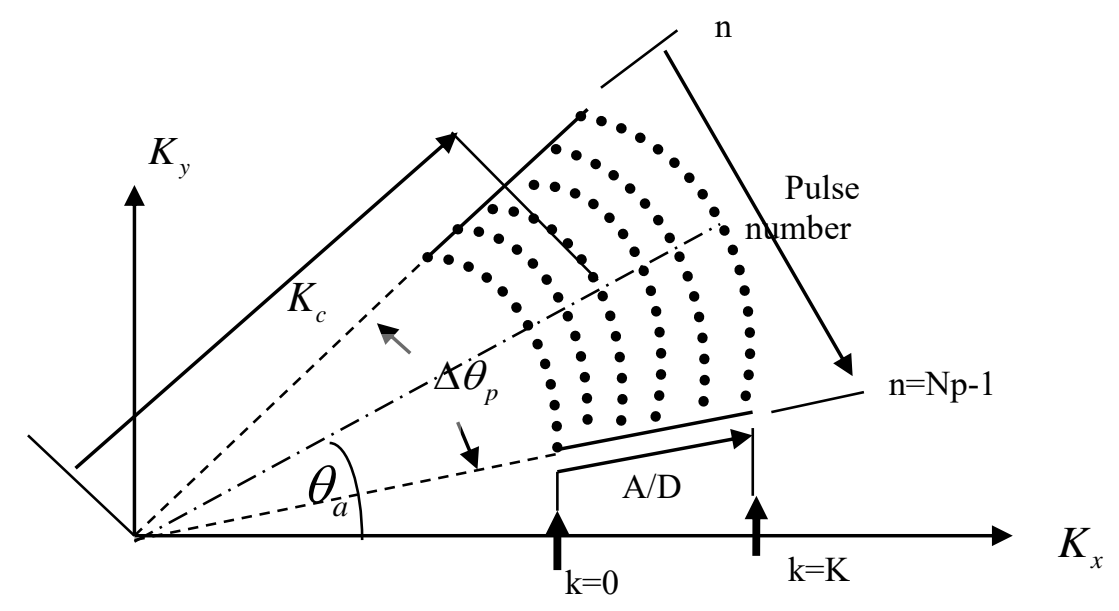

Fig. 3. Sampled data geometry in the spatial frequencies $K_{x}$ and $K_{y}$ plane

\section{Two-Dimensional Block Segmentation}

When segmenting a data matrix in 2-D, both dimensions of the data A are segmented to form block segments. The technique of segmentation is based on 2-D block mapping [11], in which the process on the $i^{t h}$ and $j^{\text {th }}$ column on a computer grid of size $\sqrt{N_{p c}} \times \sqrt{N_{p c}}$, is referred to as $P_{i j}$.

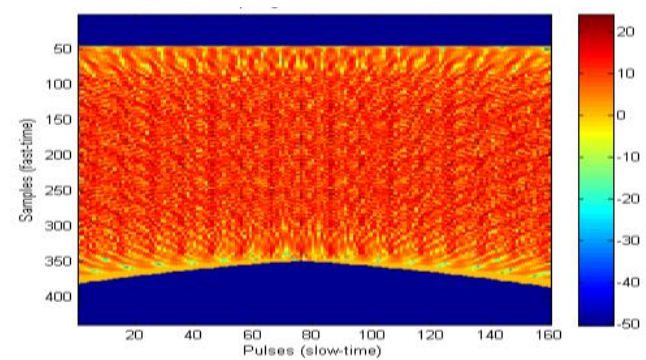

Fig.4. Spotlight SAR Returns (MathsWorks) 
Process $P_{i j}$ is assigned a sub-block of A whose upper left vertex is $\left((i-1) n / \sqrt{N_{p c}}+1,(\mathrm{j}-1) n / \sqrt{N_{p c}}+1\right)$ and whose lower right vertex is $\left(i n / \sqrt{N_{p c}}, \mathrm{j} n / \sqrt{N_{p c}}\right)$ for square data of size $n \times n$. The technique is to keep track of these vertices for every $k^{\text {th }}$ iteration of the algorithm. Each process updates its part of the block matrix during each iteration. For non-square data of size $m \times n$ the upper left vertex is represented by $\left((i-1) \mathrm{m} / \sqrt{N_{p c}}+1,(\mathrm{j}-1) n / \sqrt{N_{p c}}+1\right)$ and the lower right vertex is $\left(\mathrm{im} / \sqrt{N_{p c}}, \mathrm{j} n / \sqrt{N_{p c}}\right)$. For square data, it is segmented into $N_{p c}$ blocks or segments of size $\left(n / \sqrt{N_{p c}}\right) \times\left(n / \sqrt{N_{p c}}\right)$, for parallel processing and each block segment is assigned to one of the $N_{p c}$ processors [11], [12]. For data matrix of size $m \times n$, it would be segmented into $N_{p c}$ blocks of size $\left(\mathrm{m} / \sqrt{N_{p c}}\right) \times\left(n / \sqrt{N_{p c}}\right)$. Some resizing of some data prior to segmentation may also be necessary as discussed in the next chapter. Fig. 5 shows an example of a $4 \times 4$ segment grid of data A and how it is segmented in 2-D for parallel processing. Details of the steps of execution at each processor and communications requirements for the 2-D parallel algorithm are given in [13], [14], 15]. The first and second dimension segmentation can also be used to achieve 2-D segmentation as in Jagged-Like and Checkerboard methods [13].

In general, d-dimensional array, data can be segmented using up to d-dimensional block segments. Image data segmentation may be viewed as a process that partitions

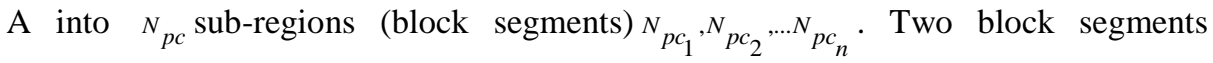
$N_{p c_{i}}$ and $N_{p c_{j}}$ would be adjacent if their union forms a connected set. If all the block segments are in the region $N_{p c_{i}}$, then they have the same intensity level and $Q\left(N_{p c_{i}}\right)=T R U E$. The algorithm below (fig. 6) was developed to parallel process the segmented SSAR data. 


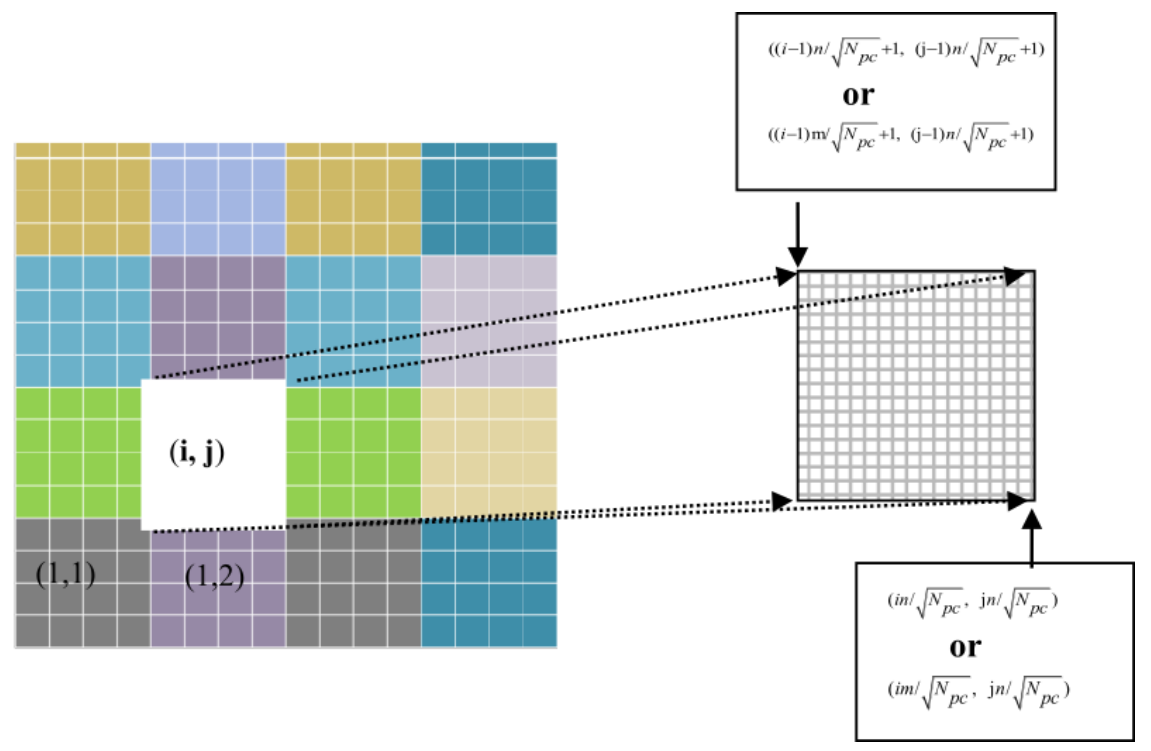

Fig. 5. Matrix A distributed by 2-D segmentation into $\sqrt{{ }_{p c} \times \sqrt{{ }^{N c}}}$ segments and the projection of $\mathbf{A}$ assigned to processor $N_{p c_{i, j}}[11]$.

The reformatted SSAR data of size 128 × 1989 was resize to $128 \times 1990$ and was segmented into 2-D segments for each processor. Each segment had to be of size $\left(\mathrm{m} / \sqrt{N_{p c}}\right) \times\left(n / \sqrt{N_{p c}}\right)$ which translated to $64 \times 995$, for each of the 4 parallel processors $(2$ $\mathrm{x} 2$ processor grid). Smaller segments could also be obtained by increasing the size of the processor grid. The square data implied that $m=n$ and segment size would be $\left(\mathrm{m} / \sqrt{N_{p c}}\right) \times\left(n / \sqrt{N_{p c}}\right)$. Table 1 shows the simulation data for 2-D Segmentation algorithm.

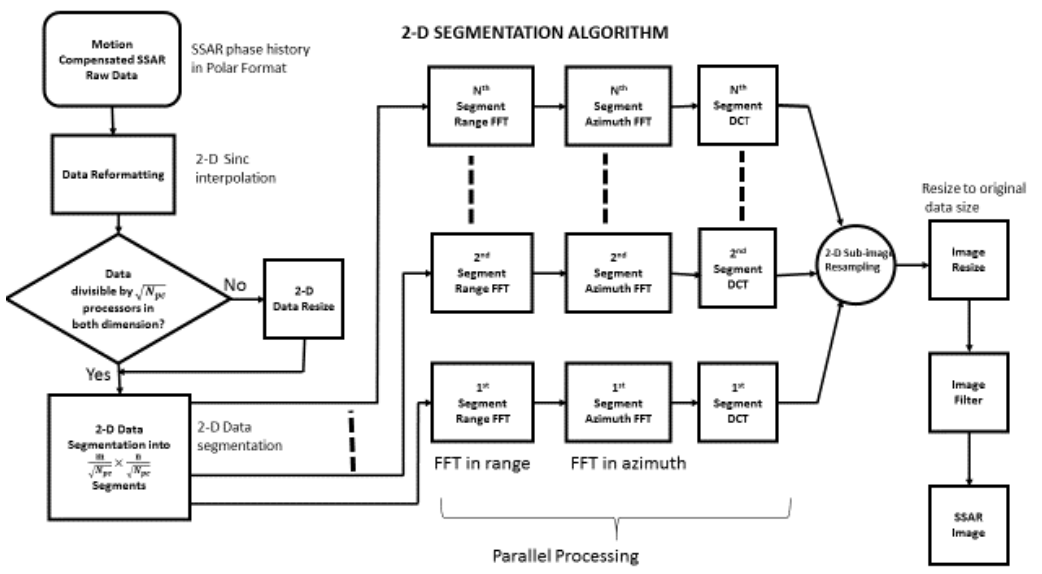

Fig.6. 2-D Segmentation Algorithm 
SAR processing creates a composite image of the ground from signals generated by a moving airborne radar platform. It is a computationally intensive process, requiring image processing and extensive input/output file exchange. Parallel processing was employed to speed up the processing of the SSAR data segments. Use of High Performance Computer could further speed up the processing of such data. The processing of the segmented SSAR data was accomplished through up-sampling, range and azimuth compression and sub-image resampling and filtering.

\section{Sub-image Reconstruction Errors}

An important factor affecting image reconstruction error and computational complexity is the size of the sub-image. Subdividing an image reduces correlation between adjacent images to some acceptable level. It also facilitates the achievement of image size $n \times n$ where $n$ is an integer power of 2 . Image compression using transform coding is then applied to reconstruct the image. The image is reconstructed from reduced amount of data required to represent an image. There are basically three techniques used to transform sub-images and these are the discrete Fourier transformation (Fast Fourier Transformation, (FFT)), the Walsh-Hadamard Transformation (WHT) and Discrete Cosine Transformation (DCT). The level of compression and computational complexity increase with sub-image size. Reconstruction errors tend to decrease as the sub-image size increases. The impact of sub-image size on transform reconstruction error for FFT, WHT and DCT is shown in fig.7. The three curves are the FFT, WHT and DCT error curves. It is observed that larger size sub-images have lower root-mean square error values when compared with small size sub-images.

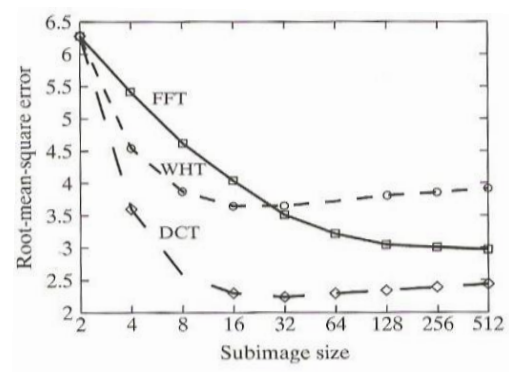

Fig.7. Reconstruction errors versus sub-image size [5]

The three curve are seen to intersect at sub-image size $2 \times 2$ with the highest root-mean square error value. When the efficiencies of FFT, WHT and DCT in sub-image processing are analysed, it is observed that DCT is a more efficient technique despite an increase in sub-image size.

\section{Results Analysis}

In this research, we combined FFT and DCT to reconstruct a resultant image from 
sub-images with the least error. For a six point target SSAR scene, we show resultant image after sub-image recombination without DCT (fig. 8) and result with DCT (figs. $9 \& 10)$. The weakness of using FFT only is that the resultant image resolution is poor. It can be observed also that apart from poor image resolution, the targets of FFT processed segments appear distorted and out of sync with the true target locations. FFT processing of segments causes the resultant sub-images to shift position before recombination as shown in fig. 8 .
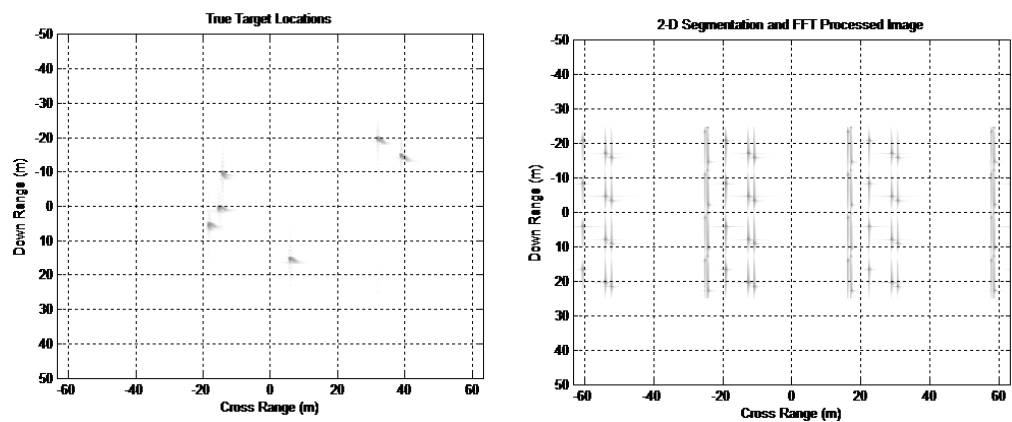

$\begin{array}{lll}\text { (a) True target locations } & \text { (b) Location of targets of FFT sub-image processing }\end{array}$ Fig. 8. Sub-image reconstruction errors with FFT

To reduce these reconstruction errors, the algorithm implemented both FFT and DCT and the results are shown in fig. 9 and 10. We show results of both noise free and noisy environment.

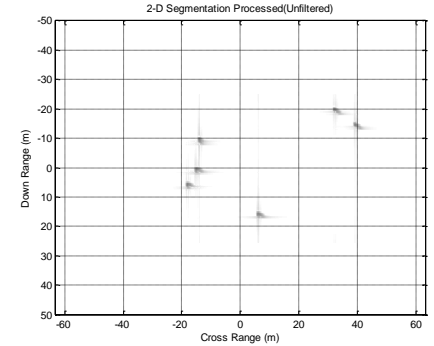

(a) Gray scale Image

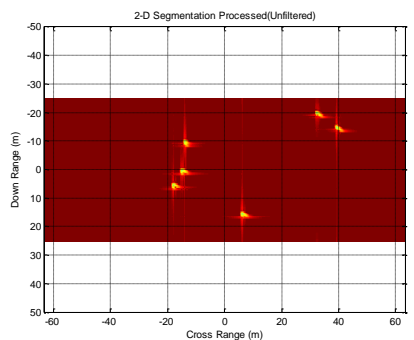

(b) Full colour map

Fig. 9. Noise free image of 6 point target scene

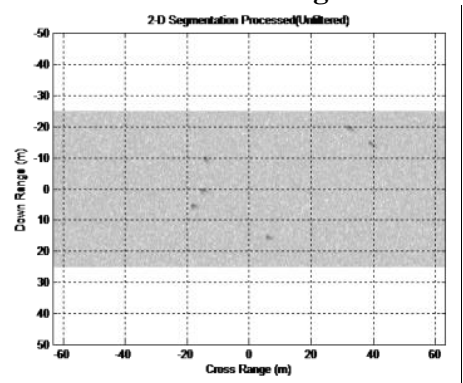

(a) Gray scale image

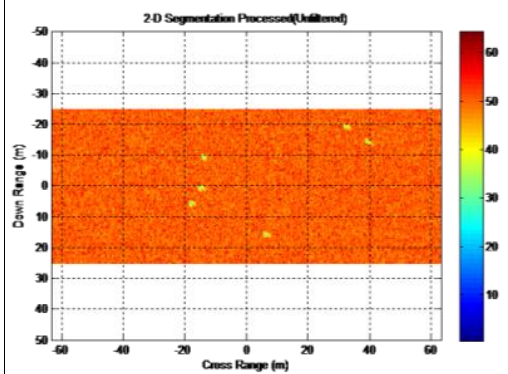

(b) Full colour map

Fig.10. Noisy image of 6 point target scene 
In fig. 11, the reconstruction of a 36 point target scene using FFT and DCT is shown for noise free environment.

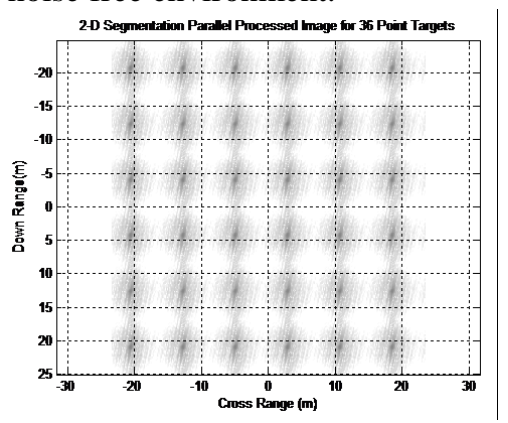

(a) Gray scale image

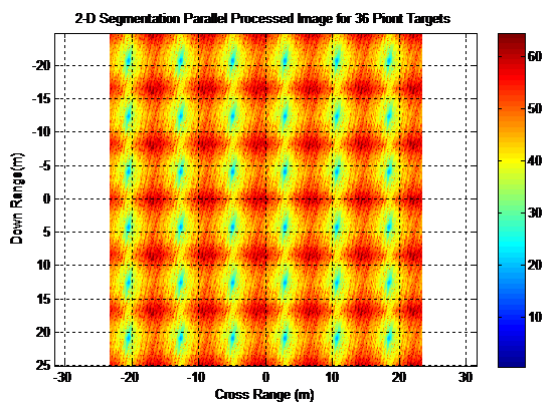

(b) Full colour map image

Fig. 11. 2-D Segmentation Image for 36 Point Target Grid

\section{Summary}

In this paper, non-traditional techniques (1-D and 2-D block segmentation) for segmenting SSAR data and images were presented. While the traditional segmentation techniques are based on certain characteristics such as intensity, amplitude, texture etc., non-traditional techniques may or may not depend on characteristics depending on the application. Segmentation of the radar data and its parallel processing addresses the limitation of memory and processing speed. However, the recombination of processed segments reduces the image quality due to recombination errors. The impact of sub-image size on reconstruction error when using image compression as a reconstruction technique was presented. It was observed in this research that the larger the sub-image size, the smaller the will be the reconstruction errors. The use of both Fast Fourier transformation (FFT) and Discrete Cosine transformation (DCT) in SSAR data processing reduces the errors associated with sub-image reconstruction.

\section{References}

[1]. Mahafza, B. R.: MATLAB Simulations for Radar Systems Design. Decibel Research, Inc, Huntsville, Alabama, Chapman \&Hall/CRC (2004).

[2]. Jakawatz, C.V. Jr., Wahl, D. E., Elchet, P. H., Chiglia, D. C., and Thompson, P.: ASpotlight-Mode Synthetic Aperture Radar: A Signal Processing Approach, Sandia National Laboratories, Albuquerque, New Mexico, Boston, London, Dordrecht, Kluwer Academic Publishers.(1996).

[3]. Goodman, R. and Carrara, W.: Synthetic Aperture Radar Algorithms. In Handbook of Image and Video Processing; Edited by Bovik, A. L., San Diego, Academic Press (2000).

[4]. Carrara, W. G., Goodman, R. S. and Majewski, R. M.: Spotlight Synthetic Aperture Radar Signal Processing Algorithms. Boston, London, Artec House (1995).

[5]. Gonzalez, R. C. and Woods, R.E. (2008). Digital Image Processing, 3rd Ed, Pearson International Edition, Prentice Hall, New Jersey. 
[6]. Ochi M. K.:Applied Probability and Stochastic Processes in Engineering and Physical Sciences, John Wiley \& Sons Inc, Singapore (1990).

[7]. Salivahanan, S., Vallavaraji, A. and Gnanapriya, C. (2004). Digital Signal Processing, 3rd rpt, New Dheli, Tata McGraw-Hill Publishing Company Limited.

[8]. Bose, T.: Digital Signal and Image Processing. Utah State University, John Wiley and sons, Inc. P.R. China, Higher Education Press (2004).

[9]. Oppenheim, A. V. and Schafer, R. W.: Digital Signal Processing. Eaglewood Cliffs, New Jersey, Prentice-Hall, Inc. (1975).

[10]. Seul, M., O'Gorman, L. and Sammon, M. J.: Practical Algorithms for Image Analysis; Description, Examples and Code. Cambridge University Press, New Jersey (2000).

[11]. Grama, A., Gupta, A., Karypis, G. and Kumar V.: Introduction to Parallel Computing, 2nd Ed, Addison-Wesley, Pearson Education, New York (2003).

[12]. Mitchell, R. L.: Radar Signal Simulation. Mark Resources, Inc. Marina Del Rey, California, Artech House, Inc. (1975).

[13]. Ucar, B., Catalyurek, U.V. and Aykanat.: CA matrix partitioning interface to $\mathrm{PaToH}$ in MATLAB, Department of Biomedical Informatics and Department of Electrical and Computer Engineering, The Ohio State University, Columbus, Ohio, USA (2009).

[14]. Ucar, B. and Aykanat, C.: Encapsulating multiple communication-cost metrics in partitioning sparse rectangular matrices for parallel matrix-vector multiplies, SIAM Journal on Scientific Computing, 25 (6) (2004).

[15]. Ucar, B. and Aykanat, C.: A library for parallel sparse matrix-vector multiplies, Tech. Rep. BU-CE-0506, Department of Computer Engineering, Bilkent University, Ankara, Turkey (2005). 